\title{
Simulating Weed Propagation Via Hierarchical, Patch-Based Cellular Automata
}

\author{
Adam G. Dunn and Jonathan D. Majer \\ Alcoa Research Centre for Stronger Communities and \\ Department of Environmental Biology, Curtin University of Technology, \\ Western Australia \\ $\{$ A.Dunn, J.Majer\}@curtin.edu.au
}

\begin{abstract}
Ecological systems are complex systems that feature heterogeneity at a number of spatial scales. Modelling weed propagation is difficult because local interactions are unpredictable, yet responsible for global patterns. A patch-based and hierarchical cellular automaton using probabilistic connections suits the nature of environmental weed dispersal mechanisms. In the presented model, weed dispersal mechanisms, including human disturbance and dispersal by fauna, are approximated by pathways through a network of cells. The results of simulations provide evidence that the method is suitable for modelling weed dispersal mechanisms using multiple scales of observation.
\end{abstract}

Keywords: Environmental weeds, hierarchical patch dynamics, cellular automata, multiscale heterogeneity

\section{Introduction and Context}

Weed establishment and spread is a significant issue in Western Australia (WA), especially in the region that is known as an international biodiversity hotspot [1]. Biodiversity describes the state of an ecosystem in terms of the natural complexity through which it evolves over time. Environmental weeds are plants that degrade an ecosystem via simplification; these weeds compete more effectively than their native counterparts in an environment that is foreign to them [2. Along the south coast of WA, local and state governments, the Gondwana Link initiative [3] and other community groups benefit from a predictive analysis of the landscape-scale effects of their decisions on the propagation of weeds.

An ecological system is a complex system 4 and the intricate web of interactions between species of flora and fauna form a resilient system. A system's degradation by weeds makes it more susceptible to further degradation.

Weeds propagate by dispersal and are constrained by competition with other plants and by predation [5]. Significant dispersal vectors include seed-eating birds and grazing animals, human transport networks, watercourses, wind and agricultural disturbances. Each of these dispersal vectors acts in a different manner and often at different spatial and temporal scales. 
The approach to modelling the landscape described here is novel because it extends the cellular automata formalism to create a cellular space that is hierarchical and irregular. As a result, the structure captures the patchy nature of the landscape realistically, as well as being capable of representing GIS data with a variety of levels granularity in a single structure.

\section{Background}

Ecosystems are complex systems in which the spatial dynamics may be described by a hierarchy of patches. Existing models of spatial ecosystem dynamics vary considerably in structure; two-dimensional raster grids that discretise mechanisms of competition or propagation are typical. Hierarchical patch dynamics is a modelling hypothesis for ecosystems that matches the physical spatial structure of ecosystems and other landscape patterns, such as land use and urbanisation.

Ecological systems are heterogeneous, complex systems that are neither completely regular nor completely random [6]. They exhibit a natural patchiness [7] that is defined by the processes of dispersal and competition, and are influenced by soil structure and natural resources, fire and forestry regimes, other human disturbances and climate. Distinct and abrupt changes between vegetation type are typical (from woodland to grassland, for example) and a mosaic of vegetation types over a landscape is a natural feature of an ecological system, besides being a feature of human-modified landscapes.

Ecological systems display multiscale heterogeneity — when viewing a system at a single level of spatial granularity, the landscape may be segmented into a set of internally homogeneous patches. To be internally homogeneous, a patch need only have an aggregable set of properties that are considered to be consistent throughout the patch at that level. For example, a coarse granularity view of a landscape may be segmented into regions of forest, woodland and grassland, whereas an individual patch of forest may be segmented by the distribution of species or even by individual plants.

In the case of weed propagation, the data quality issue is one of uncertainty at the microscale. In broad terms, weed propagation is the combination of the processes of germination, competition for resources and seed dispersal, as well as the uncertain long distance dispersal (LDD) events [8]. The typical approach for modelling the dispersal of any plant is to use empirical information to build a function that aggregates the several modes of seed dispersal by likelihood for a homogeneous landscape. The 'observational reality' of weed dispersal phenomena is one of uncertainty and multiple scales. At the scale of individual plants, the effects of individual birds, other animals, water flow, or human disturbances are essentially 'random events' in the colloquial sense of the phrasing. At a slightly coarser scale, the dispersal mechanisms for weeds become more stable in the sense that a model recovers some notional sense of predictability; mechanisms are described using a radius of spread or a likely direction of spread, and this is the operational scale of weed propagation [9]. 
Given the need to capture multiscale heterogeneity and the patchiness of ecological systems, the hierarchical patch dynamics formalism [1011] may be used to model weed propagation. Hierarchical patch dynamics is a modelling method that captures the patchiness and multiscale heterogeneity of ecological systems as a hierarchy of interacting, heterogeneous (and internally homogeneous) patches.

The hierarchical patch dynamics method may be implemented as a cellular automaton [12, modified to be irregular, probabilistic and hierarchical. Coarse patches comprise several finer patches in a hierarchy. Regular-grid cellular automata introduce a bias in propagation [13, in the absence of any other method to avoid it, such as stochastic mechanisms in timing or update. Introducing stochasticity implicitly via irregularity or asynchronicity, or explicitly via a probabilistic update can restore isotropy in a cellular automata model of propagation [14. The model described in the following section includes stochasticity to match the spatial nature of an ecological landscape by following the hierarchical patch dynamics concept.

\section{Method}

The model is constructed as a network of both interlevel connections forming a hierarchy and intralevel connections forming a graph. The structure of the model captures multiple scales of observation from GIS data, relating the levels to each other via the process of abstraction. Simulations are run by traversing the structure to determine the updated state of the network, which involves discovering the likelihood of dispersal through the heterogeneous and multiscale landscapes. The approach is analogous to existing models that use dispersal curves [15] but they additionally capture the dispersal modes explicitly rather than aggregating them into a single function, and they effectively manage the multiscale heterogeneity.

A cell is defined by its state, a set of connections to its neighbourhood and its Cartesian coordinates $i, j \in \mathbb{R}$. The state of a cell $s \in \mathcal{S}$, is defined by the static physical properties taken from GIS data of a specific level of abstraction associated with its level of abstraction; $\mathcal{S}$ represents the union of the set of possible states from each level (for example, $|\mathcal{S}|=13$ in Fig. 2). The extent of a cell is defined by the Voronoi decomposition [16] of the landscape at the level to which the cell belongs and is therefore dependent on the locations of the nodes in the cell's neighbourhood. The intralevel neighbourhood of a cell is defined by the Delaunay triangulation of the subset of nodes in the graph and the interlevel neighbourhood is defined by the process of abstraction in which the hierarchy is created by physical state information.

An abstraction necessarily involves three homogeneous cells belonging to a single triangle in the Delaunay triangulation. Using this definition, the cells composed to create a new cell are guaranteed to be neighbours, they will represent an internally homogeneous environment (for the given level of abstraction) and will create a new level of abstraction in which fewer cells are used to represent 
the landscape and the average size of a cell increases. A single abstraction is illustrated in Fig. 1] The extents of the cells are given by the Voronoi decomposition and the connectivity is given by the Delaunay triangulation. In the figure, the cell $d$ is now related to cells $a, b$ and $c$ with a 'composed of' relationship and three interlevel connections are formed, contributing to the structure of the hierarchy. Note that the choice of ternary trees over other forms is the simplest choice given the triangulation method; other forms produce the same shapes using a centroid method for defining the new cell's node location.
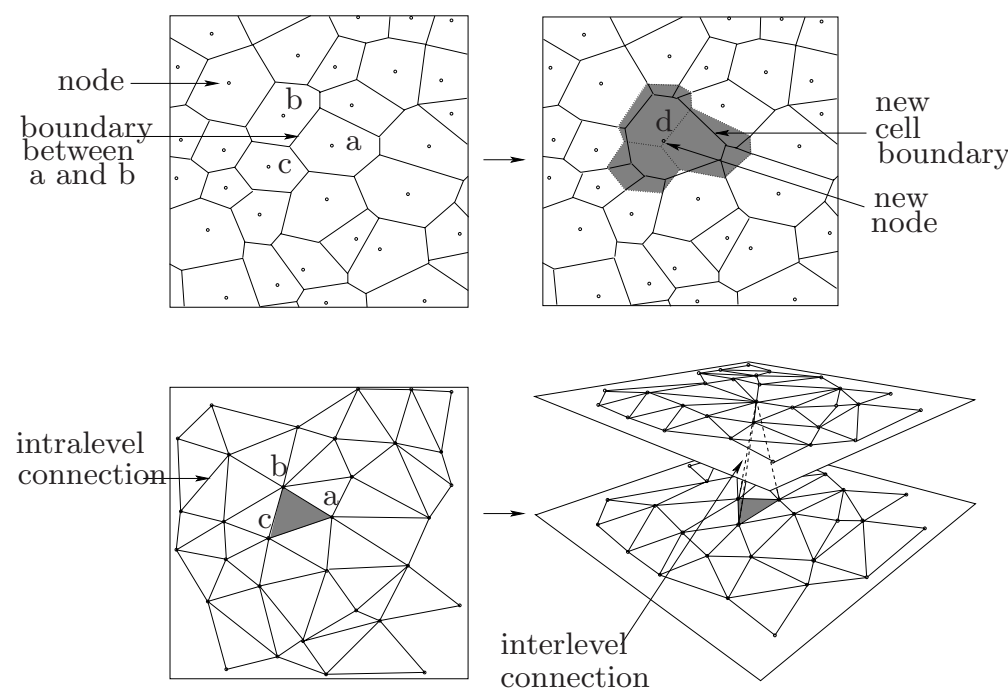

Fig. 1. A single abstraction is shown on a detail view of one landscape. The top two illustrations are the Voronoi decomposition of a set of nodes. The bottom two illustrations are a subset of the Delaunay triangulation for a set of nodes. The cells $a, b$ and $c$ in the left illustrations are abstracted to form cell $d$ as given on both of the illustrations on the right. Cell $d$ has a larger size than cells $a, b$ and $c$ (taken individually) and the distance to the nearest cells increases.

The result of multiple abstractions creates a hierarchy that builds a set of complete ternary trees. A set of points is initially distributed as a set of Halton points and each abstraction is made for a group of three internally homogeneous cells using the GIS data as a guide. The abstraction process continues until there are no more groups of homogeneous cells that may be abstracted such that the newly created distances between nodes are above a critical distance (representative of the operational scale). The above abstraction process is repeated for the several levels of GIS data until the coarsest GIS data is captured within the hierarchical structure. In Fig. 2 an example landscape is presented for for three levels of fictitious GIS data captured as raster information (see Fig. 3 for two intermediate levels of abstraction within the hierarchical structure that is built for this data). 

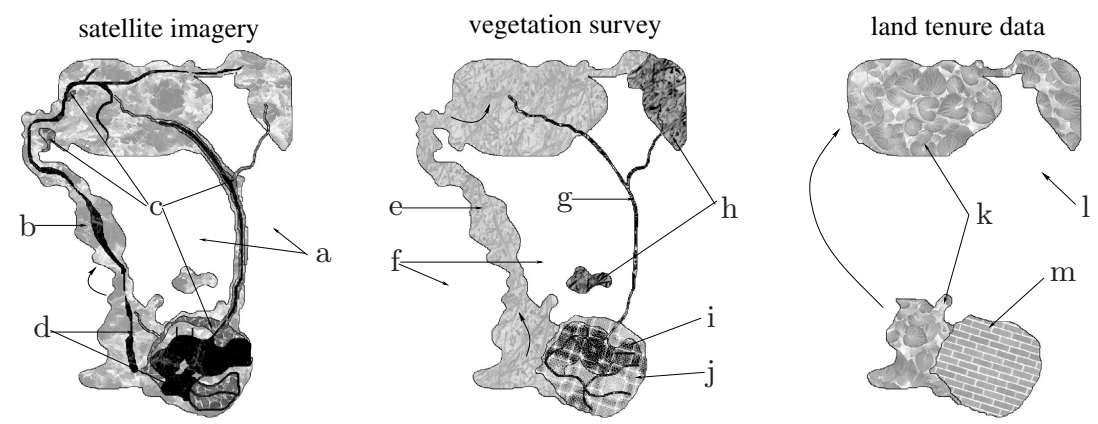

Fig. 2. Fictitious satellite imagery, vegetation survey data and land tenure data of the same landscape. Three levels of an example landscape are illustrated with the following categorisations: $a$ pasture and crops, $b$ natural vegetation, $c$ bare or disturbed, $d$ impermeable or built up, $e$ type 1 vegetation, $f$ crop or pasture, $g$ road, $h$ type 2 vegetation $i$ urban, $j$ peri-urban, $k$ nature reserve, $l$ private agricultural, $m$ private residential.

The structure of the landscape model provides a useful multiscale description of the environment for multiple scales of observation (based on GIS data) but it does not, alone, provide a method for simulating weed propagation for the variety of dispersal modes, each with their own operational scale. The simulation method chosen for the implementation described here is to associate a probability with each of the connections in the network - the interlevel hierarchy and intralevel graphs combined. At each time step (a typical synchronous timing mechanism is adopted) and for each cell, a breadth-first search through the network is used to discover the avenues of possible dispersal and the likelihood value. This path mimics the seed dispersal curves and offers a unique approach to modelling LDD via its inherent uncertainty.

Probabilities for each of the connections are determined by the state information of the connected cells and the distance between their nodes. For example, given the higher density of seed rain (dispersal by seed-eating birds perching on trees) near the periphery of remnant vegetation [17] the likelihood of dispersal between remnant vegetation and most other types of landscape is given a higher value than between two remnant vegetation cells at the vegetation survey scale. The operational scale here has a finer granularity than human dispersal modes, which occur over both longer distances and with higher uncertainty. This is analogous to dispersal curve models of short and LDD.

There are three significant assumptions made about the landscape and the process of dispersal for the implementation described here. Firstly, it is assumed that several processes including dispersal, seed predation, germination, seasonal variance and seed bank dynamics may be aggregated into a single dispersal mechanism. Secondly, it is assumed that the operational scale of the variety of dispersal modes exist within a reasonable expanse of orders of magnitude. Computer simulation would be too computation-intensive if the operational scales included millimetres and hundreds of kilometres (and their associated time scales). Lastly, 
it is assumed for these experiments that the landscape is static except for the weed propagation - ceteris paribus has been invoked in a heavy fashion for this experiment.

\section{Results and Discussion}

In testing this implementation of hierarchical patch dynamics, the results of simulations demonstrate that the method is sensitive to the choice of granularity, but that the methodology is capable of representing the multiple dispersal mechanisms and the dynamics of a weed population. Importantly, the simulations demonstrate that there is advantage in the rigorous mapping between the operational scales of the dispersal phenomena and the observational scales captured by the GIS data. The approach is useful specifically because it captures dispersal modes and multiple scales explicitly, combining them in a framework that provides the aforementioned rigorous mapping.

The results of one simulation are presented in Fig. 3 - the simulation uses the fictitious GIS data depicted in Fig. 2. In this example, an environmental weed is given properties that are typical of a fruit-bearing weed whose dispersal is dependent on birds, small mammals and human disturbance routines. A weed of this type is likely to spread along the periphery of remnant vegetation because of the desirability of the perching trees for birds in these regions, and be found more commonly in and near regions with higher populations and greater frequencies of disturbance. The probabilities associated with remnant vegetation $\leftrightarrow$ crop/pasture are relatively high, crop/pasture $\leftrightarrow$ crop/pasture are zero and any connections within or between frequently disturbed patches are associated with relatively high probabilities. Since the weed and the landscape are both fictitious, the results of the experiment are not easily verified; instead this experiment represents a proof of concept for the modelling formalism and represents a typical set of operational scales that a model may be expected to represent.

Experiments in both homogeneous and heterogeneous landscapes suggest two specific issues with the specific implementation of aggregation/abstraction. The method does not exhibit the same bias caused by a regular lattice [13] but there is an apparent degradation in the approximation to isotropy in homogeneous experiments where the highest density of points is too coarse. In simpler terms, a minimum density of points is required to maintain isotropic propagation. An issue apparent in the heterogeneous experiments is that thin corridors through which weeds may spread are sensitive to maximum densities; a model structure must represent the thinnest corridor through which a weed may propagate. It is therefore important to accurately capture the grain and extent of the operational scale - for example, some fauna involved in the dispersal may ignore thin corridors, whilst other fauna may actively use thin corridors.

By combining different forms of data (such as vegetation surveys and land tenure information), the model captures the effects of a range of dispersal mechanisms in the same simulation, predicting the combined effects of human and 

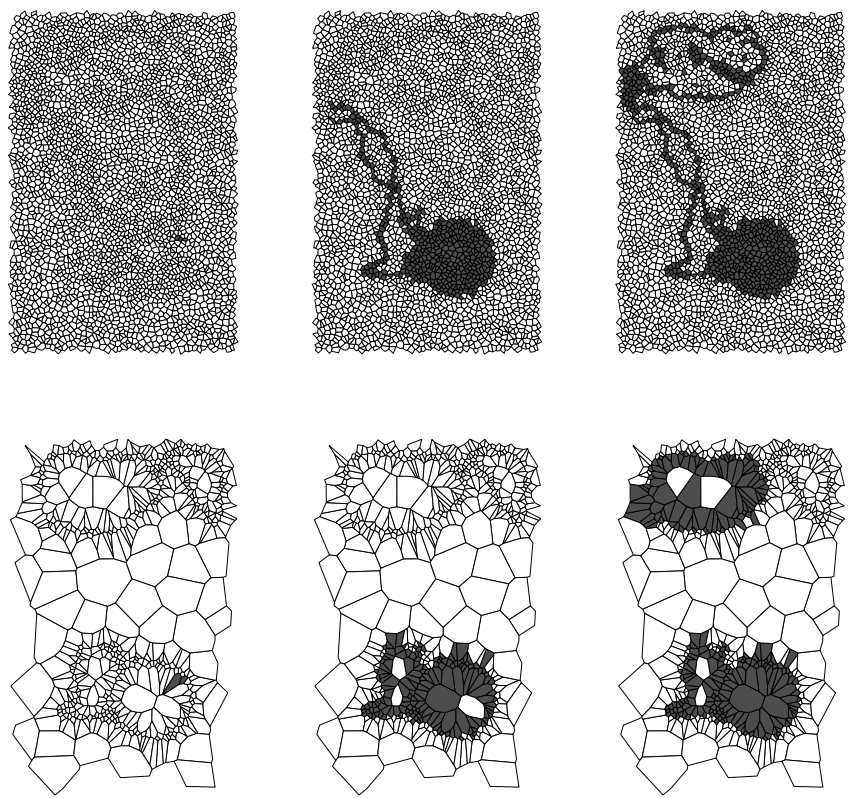

Fig. 3. The extent of cells depicted for three intermediate time steps and two levels of abstraction in the hierarchy. Elapsed time increases from left to right, the three sub-figures above are for the satellite imagery level of abstraction and the bottom three sub-figures are for the land tenure data level of abstraction. The increased rate of spread along the periphery is apparent in the top sub-figures and the dispersal over a disconnection is apparent in the bottom sub-figures.

natural dispersal mechanisms. A final assessment suggests that a more rigorous method for mapping from the dispersal mechanisms to the likelihood values is required. Specifically, the behaviour of the system is sensitive to the choice of both aggregation values between levels of abstraction and intralevel connection probabilities.

By creating a spatial model of the ecosystem in the south-west of WA using this implementation of hierarchical patch dynamics, predictive analysis of risk may be undertaken using spatial information about government policy and community group choices. Hierarchical patch dynamics is used as a model of multiscale ecological heterogeneity and the simulation presented here demonstrates a rigorous implementation of hierarchical patch dynamics. The fundamental approach to combining a variety of levels of abstraction (for human observation as well as organism perception) is the key advantage of using this approach over existing approaches.

The results of simulations suggest that the methodology is useful in creating a physically realistic (and therefore useful) model of the uncertain phenomena of weed propagation, but it is also suggestive of the need for introducing stronger links to a priori information about individual dispersal mechanisms in practical 
solutions to the issue of environmental weeds, rather than adopting a purely empirical approach.

Acknowledgments. The authors acknowledge the Alcoa Foundation's Sustainability \& Conservation Fellowship Program for funding the postdoctoral fellowship of the senior author (http://strongercommunities.curtin.edu.au) and two anonymous reviewers for helpful comments.

\section{References}

1. Myers, N., Mittermeier, R.A., Mittermeier, C.G., Fonseca, G.A.B.d., Kent, J.: Biodiversity hotspots for conservation priorities. Nature 403 (2000) 853-858

2. Ellis, A., Sutton, D., Knight, J., eds.: State of the Environment Report Western Australia draft 2006. Environmental Protection Authority (2006)

3. Anon.: Gondwana Link Website (2006) [online] http://www.gondwanalink.org, last accessed 05/10/2006.

4. Bradbury, R.H., Green, D.G., Snoad, N.: Are ecosystems complex systems? In Bossomaier, T.R.G., Green, D.G., eds.: Complex Systems. Cambridge University Press, Cambridge (2000) 339-365

5. van Groenendael, J.M.: Patchy distribution of weeds and some implications for modelling population dynamics: a short literature review. Weed Research 28 (1988) 437-441

6. Green, D., Klomp, N., Rimmington, G., Sadedin, S.: Complexity in Landscape Ecology, Landscape Series. Volume 4. Springer (2006)

7. Greig-Smith, P.: Pattern in vegetation. Journal of Ecology 67 (1979) 755-779

8. Nathan, R., Perry, G., Cronin, J.T., Strand, A.E., Cain, M.L.: Methods for estimating long-distance dispersal. Oikos 103 (2003) 261-273

9. $\mathrm{Wu}, \mathrm{J} .:$ Effects of changing scale on landscape pattern analysis: scaling relations. Landscape Ecology 19 (2004) 125 - 138

10. Wu, J.: From balance-of-nature to hierarchical patch dynamics: a paradigm shift in ecology. Q. Rev. Biol. 70 (1995) 439-466

11. Wu, J., David, J.L.: A spatially explicit hierarchical approach to modeling complex ecological systems: theory and applications. Ecological Modelling 153 (2002) 7-26

12. Chopard, B., Droz, M.: Cellular Automata Modeling of Physical Systems. Monographs and Texts in Statistical Physics. Cambridge University Press (1998)

13. O'Regan, W., Kourtz, P., Nozaki, S.: Bias in the contagion analog to fire spread. Forest Science 22 (1976) 61-68

14. Schönfisch, B.: Anisotropy in cellular automata. BioSystems 41 (1997) 29-41

15. Higgins, S.I., Richardson, D.M.: Predicting plant migration rates in a changing world: The role of long-distance dispersal. The American Naturalist 153(5) (1999) 464-475

16. Okabe, A., Boots, B., Sugihara, K.: Spatial Tessellations - Concepts and Applications of Voronoi Diagrams. John Wiley \& Sons (1992)

17. Buckley, Y.M., Anderson, S., Catterall, C.P., Corlett, R.T., Engel, T., Gosper, C.R., Nathan, R., Richardson, D.M., Setter, M., Spiegel, O., Vivian-Smith, G., Voigt, F.A., Weir, J.E.S., Westcott, D.A.: Management of plant invasions mediated by frugivore interactions. Journal of Applied Ecology 43 (2006) 848-857 